\title{
Une innovation en recherche partenariale : le cas de l'ARUC en économie sociale
}

\author{
Nisha Sajnani et Marguerite Mendell \\ Université Concordia
}

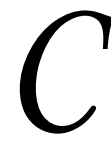
et article traitera de l'Alliance de recherche université-communauté en économie sociale (ARUC-ÉS), un bel exemple d'innovation en recherche entreprenariale au Québec. Un questionnaire a été développé pour recueillir auprès des membres de son comité de coordination leurs perceptions préliminaires concernant la phase 1 de l'organisme (2000-2005). Les résultats constituent un point de départ intéressant à partir duquel il sera possible d'esquisser les avantages et les faiblesses de la recherche entrepreneuriale. L'article comporte quatre sections. La première est une introduction concep-

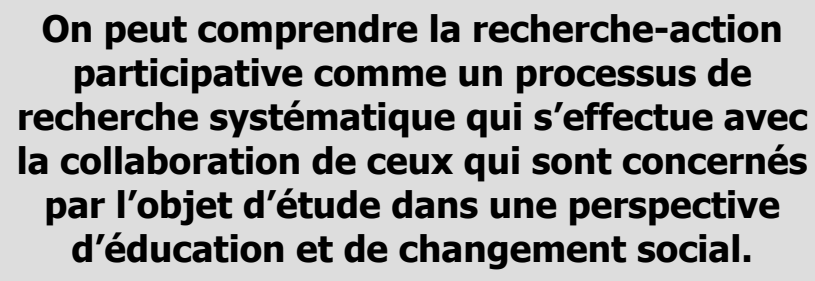
participative comme un processus de recherche systématique qui s'effectue avec la collaboration de ceux qui sont concernés par l'objet d'étude dans une perspective d'éducation et de changement social.

comme par exemple celles relatives aux systèmes, aux produits, aux procédés ou aux services.

La recherche-action participative est un type de recherche appliquée connu sous différentes appellations. La terminologie s'est développée selon les disciplines pour mettre en évidence la nature spécifique de ce type de recherche en comparaison avec la recherche traditionnelle. Parmi les termes les plus fréquemment utilisés, on verra aussi : recherche partenariale, recherche-action et recherche participative.

On peut comprendre la tuelle à la recherche-action participative pour permettre de situer l'expérience de l'ARUC-ÉS dans son contexte. Les deuxième et troisième sections présentent l'action de l'ARUC à proprement parler et le processus d'évaluation des actions menées lors de sa première phase, celle que nous étudions ici, et les propositions qui en ont résulté et qui expliquent les changements introduits pour le démarrage de la phase 2. La dernière section décrit les principaux résultats qui se dégagent des réponses au questionnaire.

\section{La recherche participative}

Par nature, la recherche en science sociale est soit fondamentale, soit appliquée. L'objectif de la recherche fondamentale est la création de connaissances nouvelles ou une meilleure compréhension du sujet à l'étude, sans intention, a priori, d'application spécifique. Pour ce qui est de la recherche appliquée, elle a pour but de développer des connaissances ou une meilleure compréhension dans un objectif de combler un besoin spécifique préalablement identifié ou de résoudre un problème particulier. De telles recherches incluent les enquêtes qui ont des objectifs précis, recherche-action participative comme un processus de recherche systématique qui s'effectue avec la collaboration de ceux qui sont concernés par l'objet d'étude dans une perspective d'éducation et de changement social. Cela implique une approche cognitive et de transformation qui regroupe quatre activités : la participation de la communauté, la recherche, l'éducation et l'action. Cette approche de recherche a pris forme à partir du développement communautaire et des programmes de formation aux adultes dans des endroits où les populations, confrontées à la domination et à l'oppression, cherchaient des solutions à leurs problèmes. Les premières références documentées de cette approche concernent des communautés dans des pays autoritaires qui l'ont utilisée pour faire contrepoids aux pratiques de recherche colonialiste et néo-colonialiste. Les travaux de Paulo Freire ${ }^{1}$ sur la prise en charge par les communautés au Brésil et au Chili ainsi que ceux de Orlando Fals-Borda en Colombie sont bien connus sur le sujet. Les expériences africaines, asiatiques et latino-américaines dans le domaine de la recherche participative sont aussi bien documentées ${ }^{2}$. La notion de recherche-action est apparue à la suite des travaux de Kurt Lewin qui l'a considérée comme 
un outil de changement dans un contexte où l'utilitarisme gagnait en popularité aux États-Unis dans les secteurs industriels, de l'éducation et du développement communautaire ${ }^{3}$.

\section{L'Alliance de recherche université- comunauté (ARUC) en économie sociale}

Le Québec a joué un rôle important dans le développement d'un programme fédéral pour promouvoir la collaboration en recherche innovatrice. Au Québec, la recherche-action a une longue histoire bien établie qui remonte aux années 1950. Elle se présentait alors sous la forme de petites collaborations expérimentales entre des chercheurs et des acteurs sociaux. Durant les trente années suivantes, divers partenariats de recherche ont été établis pour mettre ensemble chercheurs et représentants des organismes d'économie sociale. Par exemple, l'Université de Sherbrooke a soutenu un programme de recherche et développement en économie sociale et l'Université du Québec à Montréal (UQAM) a mis sur pied le Service aux collectivités pour développer des liens entre le milieu académique, les organismes communautaires, les mouvements sociaux et les organismes culturels. Jean-Marc Fontan suggère que cette évolution de la recherche-action, ou ce qu'on appelle la recherche partenariale, «permet la production des recherches thématiques et sectorielles sur différents mouvements sociaux dont le mouvement syndical, le mouvement communautaire, le mouvement coopératif ou le mouvement féministe $»^{4}$. Entre 1980 et 2000 , les deux paliers de gouvernement, provincial et fédéral, ont commencé à financer la recherche concernant l'économie sociale. L'expérience québécoise en recherche partenariale entre chercheurs et praticiens a incité le Conseil de recherche en sciences humaines (CRSH) du Canada à développer un programme national de collaboration, un programme qui reposait d'abord sur la communauté et qui visait diverses applications de partenariats de recherche. En 2000, il a mis sur pied le programme «Alliance de recherche Université-Communauté » (ARUC) dans le but «d'appuyer la création d'alliances entre les universités et les communautés qui, par un processus axé sur la collaboration continue et l'apprentissage mutuel, favoriseront la recherche novatrice, la formation et la création de nouvelles connaissances $»^{5}$.

L'approche de l'ARUC impose aux chercheurs et aux partenaires communautaires des méthodes de recher- che très innovantes et participatives pour s'attaquer à des préoccupations particulières dans les domaines sociaux, culturels ou économiques. Le mandat de l'ARUC de générer de nouvelles connaissances instrumentales, interactives et critiques s'appuie sur la recherche collaborative qui reconnaît l'importance de ce qu'on appelle la co-construction $d u$ savoir. Plus qu'une simple méthodologie orientée vers la résolution de problèmes, le programme ARUC du CRSH s'est donné les objectifs spécifiques suivants :

- favoriser l'échange de connaissances, de ressources et de compétences entre les universités et les organismes de la communauté;

- enrichir la recherche, les méthodes d'enseignement et les programmes des universités;

- renforcer la capacité des collectivités de prendre des décisions et de résoudre des problèmes;

- rehausser la formation et l'employabilité des étudiants en leur donnant diverses possibilités d'acquérir des connaissances, des compétences et des qualités professionnelles grâce à des travaux de recherche pratiques et à une expérience connexe.

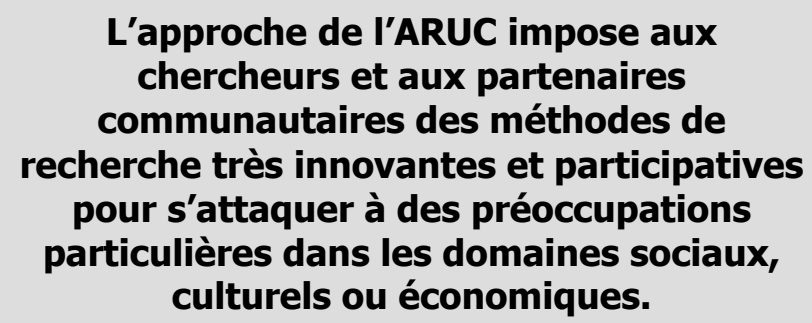

Les chercheurs et praticiens québécois ont accueilli avec enthousiasme la création de l'ARUC en économie sociale dès la création de la première phase du programme du CRSH. Il s'agissait du premier centre de recherche interuniversitaire québécois entièrement dédié à la recherche partenariale en économie sociale. L'ARUC-ÉS définit ainsi la recherche-action : « La recherche-action est un objet méthodologique complexe; dans son essence même, elle est au moins quadruple : elle est recherche appliquée, recherche impliquée, recherche imbriquée et recherche engagée. » « Il s'agit de recherches dans lesquelles il y a une action délibérée de transformation de la réalité; recherches ayant un double objectif : transformer la réalité et produire des connaissances concernant ces transformations. » « La recherche-action débouche sur une nou- 
velle posture et une nouvelle inscription du chercheur dans la société, par la reconnaissance d'une compétence à la recherche de praticiens du social. »

Dans un premier temps, l'ARUC-ÉS regroupait quatre universités : l'Université du Québec à Montréal (UQAM), l'Université du Québec en Outaouais (UQO), l'Université du Québec à Chicoutimi (UQAC) et l'Université Concordia. Plus de 30 chercheurs ont été impliqués lors de cette première phase d'implantation. L'ARUC a aussi compté sur six organisations québécoises en économie sociale, soit :

- le Chantier de l'économie sociale;

- la Confédération des syndicats nationaux (CSN) et la Fédération des travailleurs et travailleuses du Québec (FTQ);

- les deux fonds de travailleurs, FondAction - le fonds de développement de la CSN pour la coopération et l'emploi -, et le Fonds de solidarité, de la FTQ;

- le Réseau d'investissement social du Québec (RISQ).

\section{L'esprit de partenariat qui anime I'ARUC se fait aussi sentir dans son mode de gouvernance.}

Dans sa forme originale, l'ARUC-ES était structurée en neuf chantiers thématiques appelés chantiers d'activités partenariales (CAP). Chaque chantier était coprésidé par un chercheur et par un partenaire du milieu. Ces CAP étaient les suivants :

- développement local et régional;

- développement durable;

- financement;

- maintien de l'emploi et insertion socioprofessionnelle ;

- logement communautaire;

- services aux personnes;

- loisirs et tourisme social;

- évaluation sociale et systèmes d'information;

- échanges et comparaisons internationales.
Denis Bussières et Jean-Marc Fontan présentent ces groupes de travail comme étant «à la fois des lieux d'échange et de réflexion, des lieux de diffusion des résultats de recherche et des lieux de production de nouvelles recherches $\gg{ }^{6}$. Ces chantiers ont donc procuré un espace pour mettre en commun le savoir théorique et le savoir pratique et pour établir un dialogue continu entre les chercheurs et les praticiens.

L'esprit de partenariat qui anime l'ARUC se fait aussi sentir dans son mode de gouvernance. Un comité de direction, formé des co-directeurs des neuf CAP, est en quelque sorte le «conseil d'administration » et voit à l'orientation générale de l'organisme. Ce comité de synthèse établit également des passerelles entre les différents CAPs et, de cette façon, permet une bonne représentativité de l'économie sociale par ses différents secteurs d'activités, ses mouvements sociaux et les outils de développement ainsi que les politiques transversales qui lui sont propres. De fait, c'est l'économie sociale qui a initié ce besoin pour un nouvel environnement de recherche où peuvent être étudiés ces divers enjeux et, ainsi, elle a contribué à innover dans un modèle de recherche qui allie l'interdisciplinarité et la cohabitation de la recherche fondamentale, appliquée et partenariale.

Bussières et Fontan, deux membres du comité de coordination, ont produit un document de réflexion sur la première phase d'activités de l'ARUC-ÉS en commençant par une description de l'organisme et de ses trois objectifs principaux :

- produire des savoirs neufs qui seront utiles au développement des collectivités. Ceci implique la construction d'un savoir sur l'évolution de la nouvelle économie sociale au Québec et ailleurs;

- partager les connaissances entre les universités et les communautés, mais privilégier un transfert dynamique et bidirectionnel;

- soutenir le développement du modèle québécois d'économie sociale comme système d'innovation de l'économie sociale.

Il est important de souligner que, parallèlement à ces objectifs destinés à répondre aux besoins des organisations d'économie sociale, il est aussi fondamental pour l'ARUC-ÉS de remplir son rôle d'analyse critique et de recherche. Cette combinaison de recherche- 
action ou de recherche partenariale et de recherche fondamentale contribue à la particularité de l'ARUCÉS et à sa capacité d'atteindre ses objectifs.

L'ARUC-ÉS a démontré avec succès dans sa pratique sa capacité de consolider et de poursuivre son travail quand, en 2005, elle s'est vu octroyer une seconde subvention du CRSH. Dans sa deuxième phase, l'Alliance a restructuré ses activités autour de cinq chantiers : développement local et régional, financement, logement communautaire, services aux personnes et loisir et tourisme social. Les CAP (de la première phase), considérés comme transversaux, ont été intégrés à un des cinq chantiers thématiques, à l'exception du financement à cause de son évolution à la fois comme secteur (l'économie solidaire) et des besoins constants en nouveaux outils financiers nécessaires à la capitalisation de l'économie sociale.

\section{Depuis 2000, au moment où I'ARUC a été lancé, 85 projets de recherche conjoints ont été menés sur des thèmes variés.}

\section{Réflexion et évaluation de l'expérience ARUC : la transition vers la phase 2}

La reconduction du programme de l'ARUC par le CRSH a été précédée par une réflexion parmi les chercheurs et les praticiens alors qu'il fallait présenter une nouvelle soumission ${ }^{7}$. Cette réflexion s'est poursuivie pendant la phase 2 par les chercheurs et les praticiens qui passent en revue les succès et les ratés de la première phase. La question du transfert de connaissances a constitué un point central de cette réflexion non seulement parce qu'elle concerne tous les CAP, mais surtout parce qu'elle touche les représentants de l'économie sociale qui ne participent pas nécessairement aux activités de l'ARUC, si ce n'est qu'à quelques événements publics, conférences ou symposiums. Autrement dit, est-ce que les travaux de l'ARUC-ÉS réussissent à rejoindre ceux qui travaillent dans les nombreuses organisations partenaires de l'Alliance ? Jusqu'à quel point 1'ARUC a-t-elle rempli son mandat de transfert de connaissances? Quels sont les obstacles à ce transfert de connaissances ?

Durant cette période, plusieurs membres de l'ARUC étaient engagés dans un processus d'évaluation pour connaître la capacité de l'organisation de rejoindre les associations, les mouvements, les communautés et les organisations qui les représentent. Cet effort de réflexion entre les membres de l'ARUC s'est traduit par un grand nombre d'articles qui ont contribué à la réflexion sur le sujet. Il s'est dégagé trois enjeux principaux relativement à l'efficacité du transfert de connaissances par l'ARUC : la production du savoir, les outils collectifs et les conditions gagnantes. Concernant la production de la connaissance et les outils collectifs, Bussières et Fontan ont estimé à plus de 150 le nombre de chercheurs et de praticiens qui ont participé à de telles activités durant les premiers cinq ans de l'ARUC. Ils ont recensé 20 séminaires et colloques organisés par les CAPs, dont plusieurs étaient ouverts au public et auxquels plus de 1500 personnes ont participé.

Depuis 2000, au moment où l'ARUC a été lancé, 85 projets de recherche conjoints ont été menés sur des thèmes aussi variés que le financement de l'économie sociale, les conditions de travail, la relation entre les populations et l'économie sociale, pour n'en nommer que quelques-uns. Bussières et Fontan attirent l'attention sur le travail de diffusion de l'ARUC-ÉS qui a produit 80 cahiers répartis dans l'une des trois collections: Interventions, Transfert et Recherche. L'organisation a aussi élaboré un dépliant et un site Web pour s'assurer d'une bonne communication entre les partenaires et avec le public.

Cette réflexion continue qui a caractérisé l'ARUC depuis ses débuts s'est enrichie au fil des nombreuses années d'expérience et permet aujourd'hui aux personnes impliquées d'identifier les conditions optimales pour la recherche partenariale. Bussières et Fontan insistent sur l'importance d'un leadership fort de la part des partenaires qui assument la co-responsabilité des chantiers et sur des échanges francs et continus entre les participants pour assurer le succès de la démarche. Ils identifient le besoin pour les deux parties d'être associées à la définition des questions de recherche et la nécessité d'un suivi administratif pour la réalisation du plan d'action de la recherche. Finalement, ils ont déterminé qu'il doit exister différents moyens de transfert pour s'assurer d'une diffusion adéquate des résultats de recherche.

Concernant cette question du transfert de connaissances, ils proposent d'établir dès le début de la recherche les objectifs de diffusion des résultats pour 
être en mesure d'allouer à cette importante composante de la recherche partenariale les ressources nécessaires.

La réflexion actuelle menée par 1'ARUC-ÉS, alors que cette dernière entreprend sa deuxième phase, est à l'image de l'organisation, c'est-à-dire qu'elle est participative et interactive et implique plusieurs acteurs et méthodologies. Parmi celles-ci, on retrouve un questionnaire préliminaire que nous avons préparé à la toute fin de la phase 1 pour permettre à un nombre réduit de participants clés de s'exprimer sur cette expérience au moment où on s'apprête à aller de l'avant avec la phase suivante. Les résultats de ce questionnaire font ainsi partie du processus en cours qui est, quant à lui, une des particularités de l'ARUC d'évaluer de façon régulière sa capacité à atteindre ses objectifs, d'améliorer les conditions qui ont permis des succès et de s'interroger sur ses échecs et ses limites. Et cela, dans un esprit de dialogue et de saine délibération. Même s'il était relativement limité, le questionnaire a permis de dégager des éléments utiles à la réflexion en cours à l'ARUC et également pertinents sur la recherche partenariale en général. Une synthèse des réponses obtenues est présentée à la section suivante.

\section{Le questionnaire : méthodologie et résultats}

Le questionnaire a été développé avec la participation de quatre membres du comité de coordination dans le but d'être distribué à tous les co-directeurs de chacun des chantiers d'activités, ceux-là même qui forment la totalité du comité de coordination. Sur les vingt membres actifs qui forment ce comité de la première phase de l'ARUC, dix ont finalement participé à l'enquête. Quatre des répondants étaient des chercheurs académiques, alors que les six autres étaient des partenaires de la communauté. L'objectif premier du questionnaire était de déterminer si l'ARUC avait implanté un processus qui, en plus de rencontrer ses objectifs de départ, était conforme aux principes de la rechercheaction participative, lesquels doivent permettre aux participants de satisfaire leurs besoins et leurs intérêts dans leur propre milieu et de participer à la mise en place de politiques tant au niveau fédéral que provincial.

Le questionnaire était divisé en cinq sections. La première concernait les avantages et les désavantages de travailler dans le cadre de l'ARUC-ÉS. La seconde interrogeait sur l'expérience des participants et la nature de leur implication. La troisième section demandait aux participants d'évaluer le niveau d'atteinte des objectifs de l'ARUC. La quatrième section concernait les différentes formes d'évaluation disponibles à l'intérieur de l'organisation. Finalement, la cinquième section était destinée à connaître leur perception quant à la nature des retombées de l'ARUC-ÉS.

Dans la première section du questionnaire, on a demandé aux répondants de se référer à leur expérience à l'ARUC, c'est-à-dire à leur évaluation quant à l'atteinte des objectifs de l'organisation ainsi qu'aux avantages et désavantages de travailler dans le cadre du modèle de partenariat qui est le sien. En général, les répondants ont déclaré leur expérience positive et considèrent ce genre de modèle pertinent pour encourager un dialogue structuré entre les personnes et les groupes impliqués en économie sociale. On estime que l'ARUC procure un cadre et une infrastructure de financement qui permet un lien entre l'action et la recherche. Un des défis les plus fréquemment mentionnés a été la différence de culture à laquelle étaient confrontés autant les universitaires que les gens du milieu. On a fait référence par exemple à des chercheurs qui, habitués à travailler seuls, ont dû s'adapter au travail en équipe. Le langage particulier des chercheurs a aussi nécessité un apprentissage de la part de certains membres de la communauté, allant jusqu'à la frustration lorsque venait le temps de transférer les résultats de recherche longtemps attendus dans un format tangible en actions concrètes qui peuvent répondre à leurs besoins immédiats.

Les partenaires académiques ont souligné les avantages du modèle en regard de sa capacité de mobiliser des partenaires multiples, plusieurs projets et la création d'un réseau de recherche efficace. Ils ont aussi fait remarquer que l'ARUC-ÉS joue un rôle important dans le développement de la recherche innovatrice, car l'organisme est devenu une référence pour les organismes du secteur. Un répondant mentionne que " l'ARUC a permis de développer un savoir systématique sur la réalité de l'économie sociale et d'encourager le développement d'un cadre conceptuel et analytique. [...] Il a facilité la transmission du savoir auprès des membres intéressés de la communauté en gardant à l'esprit son objectif général d'influencer les politiques publiques ». Il a aussi contribué à mettre en place des programmes universitaires pertinents tout en 
créant des opportunités pour l'enseignement et la recherche en économie sociale. Pour les membres de la communauté, l'accès à un réseau de chercheurs constitue un des grands avantages de ce modèle, sans compter les ressources disponibles sur un grand nombre de sujets d'intérêt qu'ils peuvent présenter à l'intérieur des chantiers d'activités.

Dans la seconde section du questionnaire, les participants étaient invités à commenter la capacité du partenariat mis en œuvre par l'ARUC pour générer des collaborations et, si ce n'était pas le cas, à en identifier les raisons. Les réponses reçues ont majoritairement mentionné que, effectivement, il y avait beaucoup d'attention donnée à la collaboration à travers des conférences, des séminaires et de nombreuses rencontres. Toutefois, autant les chercheurs que les gens du milieu ont fait part de leurs préoccupations quant aux dépenses associées à leur participation aux activités qui s'avèrent prohibitives pour les participants qui vivent à l'extérieur de Montréal, là où se tient la majorité des activités. La vidéo conférence a été suggérée pour corriger cette situation particulière. Les chercheurs académiques ont souvent mentionné qu'une des limites du modèle était le manque de temps et de financement pour appuyer la préparation de nouvelles recherches et soutenir une meilleure participation des partenaires de la communauté.

Les gens du milieu ont estimé qu'il n'y avait pas suffisamment de contrôle sur les informations qu'ils partagent avec les chercheurs, ne sachant pas toujours comment elles sont utilisées et diffusées. Cette situation crée un malaise et affecte leur niveau de confiance et leur volonté de partager de l'information avec les chercheurs. Le transfert des connaissances est aussi un élément qui limite la participation des partenaires du milieu puisque les résultats de recherche sont produits dans un langage et une forme qui sont difficiles à saisir et à appliquer dans la réalité des organisations du milieu. Plusieurs répondants du milieu ont proposé, pour permettre aux recherches d'être mieux diffusées et éventuellement appliquées, de profiter des assemblées annuelles des organisations ou d'autres rencontres des membres pour présenter les résultats dans un format plus accessible.

Dans la troisième section, on a demandé aux participants de discuter de leur expérience de travail dans le cadre du partenariat ARUC sous l'angle de son efficacité à choisir des sujets d'intérêt et de faciliter

l'émergence de nouvelles connaissances. Plus particulièrement, on a demandé aux participants de faire une comparaison entre leur expérience dans ce modèle de recherche et d'autres expériences dans lesquelles ils ont participé dans le passé. Les réponses reçues suggèrent que les participants souhaitent poursuivre leur implication dans le modèle de partenariat proposé par l'ARUC. On y souligne les méthodes de recherche participative qui constituent un moyen efficace pour la construction de réseaux durables et pour sa capacité à combler le fossé qui sépare souvent la recherche et l'action. Les participants font ressortir la particularité de l'ARUC par le nombre de partenariats créés dans différents domaines spécialisés. Les partenaires communautaires reconnaissent le rôle déterminant de l'ARUC pour les aider à avoir une vision globale de l'économie sociale aussi bien que pour leur procurer des ressources et le langage nécessaire pour promouvoir leur image publique et leur crédibilité comme acteurs socio-économiques vis-à-vis des bailleurs de fonds et des agences gouvernementales.

Les gens du milieu ont estimé qu'il n'y
avait pas suffisamment de contrôle sur
les informations qu'ils partagent avec
les chercheurs, ne sachant pas toujours
comment elles sont utilisées et diffusées.

Les participants ont aussi été interrogés sur les modes d'évaluation en vigueur et, plus spécifiquement, on leur a demandé si le transfert de connaissances peut encourager les partenaires à poursuivre leur collaboration. Les informations recueillies indiquent qu'il n'existe pas de mécanismes formels ou explicites d'évaluation. Toutefois, les répondants mentionnent qu'il y a bien une forme d'évaluation qui s'effectue lors des rapports annuels et des rencontres du comité de coordination. Ces évaluations informelles sont considérées utiles pour amorcer des réflexions mais insuffisantes pour permettre de revoir le fonctionnement de l'organisme ou pour y introduire des changements significatifs. Quand on demande aux répondants de se prononcer sur la circulation des connaissances générées par l'ARUC, la majorité indique qu'il y a, globalement, une faiblesse à ce niveau.

Finalement, les personnes interrogées devaient s'exprimer sur les résultats de la recherche produits par l'ARUC et réfléchir sur les moyens utilisés pour permettre l'apprentissage des nouvelles connaissances et 
également sur les bénéficiaires de ces résultats. Les résultats de recherche sont diffusés au moyen de documents écrits, de présentations orales et avec des outils audiovisuels. Concrètement, on a mentionné des sites Web, des rapports, des documents de travail, des imprimés pour les médias et divers conférences conjointes ainsi que des rencontres avec des gens de la communauté comme moyens de diffusion de la recherche. En général, les répondants s'accordent pour dire qu'autant les universitaires que les partenaires de l'Alliance bénéficient des travaux effectués. Toutefois, sept répondants sur dix estiment que les universitaires bénéficient davantage des recherches à cause du manque d'attention accordé au transfert des connaissances vers la communauté. Les partenaires communautaires ont répété que les bénéfices qu'ils peuvent tirer sont directement reliés à la crédibilité accrue qui est attribuée à leur contribution aux nouvelles connaissances produites.

\section{La volonté de I'ARUC a été de rapprocher les universitaires et la communauté par la création de partenariats et la mise en place de structures administratives efficaces.}

\section{Conclusion}

Lors de sa dernière conférence internationale sur les études supérieures, l'UNESCO a reconnu que «les nouveaux modes de production de la connaissance font que les universités ne sont maintenant qu'un des acteurs parmi d'autres à l'intérieur d'un système de production de la connaissance réparti dans l'ensemble de la société [...] les universités ont besoin de revoir leurs relations publiques et de reconstruire des liens de confiance avec la société; non seulement avec le gouvernement et l'industrie, mais aussi avec les instances locales et régionales $»^{8}$.

Les pionniers de la recherche-action participative ont permis le développement de la production de la connaissance avec l'aide de nombreux partenaires depuis le début des années 1970. Cette approche de collaboration dans la production du savoir s'est aussi enracinée dans les universités québécoises et, plus tard, s'est vu reconnue par les bailleurs de fonds provinciaux et fédéraux qui ont développé et supporté les partenariats entre les universités et les communautés.
La volonté de l'ARUC a été de rapprocher les universitaires et la communauté par la création de partenariats comptant un nombre égal de chaque groupe et la mise en place de structures administratives efficaces pour permettre à la communauté de participer à la production de nouvelles connaissances et de dynamiser son milieu afin qu'elle s'engage dans sa propre vision de changement. Van Schendel explique que l'ARUC-ÉS est une forme de recherche où la diffusion, le transfert et l'apprentissage de la connaissance sont des éléments clés. Il cite Dolbec pour définir la recherche-action : «Un effort constant de relier et de mener en même temps action et réflexion, de réfléchir sur son action en vue de l'améliorer et d'agir en s'observant dans le but de développer son savoir $»{ }^{9}$. Dans le domaine de la recherche, l'ARUC représente une approche innovatrice qui est en constante évolution et qui encourage l'autoréflexion et l'autocritique tout au long du processus de production et de diffusion de la connaissance. Ce questionnaire, même limité, est une contribution aux efforts de l'ARUC dans sa volonté de tirer des leçons de la phase 1 et de réfléchir à ses orientations pour la phase 2 .

Les réponses obtenues du comité de coordination confirment leur volonté de poursuivre leur engagement dans ce modèle de partenariat. En général, les réponses suggèrent que les partenaires, autant universitaires que communautaires, ont bénéficié de cette collaboration par la création d'un espace de recherche durable qui est en mesure de répondre aux préoccupations des organisations communautaires. À partir de ce bref questionnaire, il appert que l'ARUC-ÉS a atteint ses objectifs de valorisation de l'économie sociale en créant des structures qui facilitent l'échange des connaissances entre les organismes de la communauté et les institutions de recherche.

Les répondants ont aussi parlé ouvertement des limites de ce modèle de partenariat, en particulier sur la production et le transfert des connaissances entre les partenaires académiques et ceux de la communauté. Même si les répondants reconnaissent l'importance de cette initiative, plusieurs ont indiqué que les structures sont parfois trop rigides pour permettre l'innovation dans les domaines de la création, de la diffusion et du transfert des connaissances. Ils ont signalé que les moyens utilisés pour conserver les résultats de recherche et les distribuer n'étaient pas toujours dans l'intérêt des partenaires de la communauté. Les observations faites par les répondants concernant le manque 
de temps chronique et les difficultés liées aux différences de culture entre les chercheurs et les gens du milieu rejoignent tout à fait les propos de Bussières et Fontan sur la question. Ces derniers ont noté des différences dans les objectifs entre les deux groupes de partenaires: «Pour les praticiens, la recherche vient répondre avant tout à des problématiques vécues par leur organisation ou en fonction de besoins précis [...]; pour les chercheurs, les objectifs sont de l'ordre du développement de la connaissance, du développement d'un ou de plusieurs secteurs de recherche et de la formation des étudiants qui sont associés à l'activité de recherche partenariale $\gg{ }^{10}$.

Les répondants ont insisté sur le rôle central d'une direction et d'une implication partagées pour assurer le succès de leur partenariat. Ils ont aussi souligné le besoin d'un suivi administratif dans le processus de recherche pour garantir l'atteinte des objectifs pour chacun des deux groupes. Ces éléments constituent les conditions gagnantes aux yeux de Bussières et Fontan. Les difficultés liées au transfert des connaissances ont été soulevées et constituent une préoccupation importante pour la phase 2 de l'ARUC-ÉS alors que tous les partenaires se questionnent pour y apporter des correctifs. Il aurait sûrement été utile de procéder à une distribution plus exhaustive du questionnaire; toutefois, la présente recherche s'inscrit parmi les nombreux mécanismes de réflexion qui se déroulent en continu à l'intérieur de l'ARUC, ce questionnement constant étant lui-même un processus actif d'évolution de la recherche partenariale.

\section{Notes et références}

1 Freire, P. (1972). Pedagogy of the Oppressed, London, Sheed and Ward.
2 Fals Borda.O. (1991). Knowledge and Social Movements, Santa Cruz, Merrill Publications, University of California; Kassam, Y et K. Mustafa, (1982). Participatory Research: An Emerging Methodology in Social Science Research, New Delhi, Society for Participatory Research in Asia; Tandon, R. et W. Fernandes (1981). Participatory Research and Evaluation: Experiments in Research as a Process of Liberation, New Delhi, Indian Social Institute.

3 Greenwood, D.J. et M. Levin (1998). Introduction to Action Research, Social Research for Social Change, Thousand Oaks (CA), Sage Publications.

4 Fontan, J.-M. (2005). La recherche partenariale en économie sociale : l'expérience québécoise, UQAM, p. 3.

5 Document du Conseil de recherche en sciences humaines du Canada (CRSH, 1999) consulté sur Internet le 20 juin 2006 :

http://www.sshrc.ca/web/apply/program_descriptions/cura f.asp\#1.

6 Bussières, D. et J.-M. Fontan (2005). L'expérience de recherche de l'Alliance de recherche université- communautés en économie sociale, ARUC-ÉS, p. 7.

7 Bussières, D. et J.-M. Fontan, op. cit.; Fontan, J.-M., op. cit.; Van Schendel, V. (2004). Diffusion et transfert des connaissances: quelques notes sur les enjeux et les besoins dans le cadre des projets en partenariat, ARUC-ÉS, février; Van Schendel, V., J.M. Fontan, D. Bussières et L. Proulx (2004). Développer l'arrimage et la proximité chercheurs-milieu: pistes pour une amélioration de la diffusion et du transfert à L'ARUC-ÉS, document pour la comité de coordination du 6 avril, ARUC-ÉS.

8 Cité par Van Schendel, V., op. cit., p. 12.

9 Cité par Van Schendel, V., op. cit., p. 5.

10 Bussières, D. et J.-M. Fontan, op. cit., p. 12. 